Case Report

\title{
Diagnosis of Isolated Cleft of the Anterior Mitral Leaflet in a Dog: A Case Study Using Real-Time Three- Dimensional Echocardiography
}

\author{
Ryuji Araki, ${ }^{1,2,3}$ Koji Iwanaga, ${ }^{2}$ Kazunori Ueda, ${ }^{1}$ and Mitsuhiro Isaka $\mathbb{D}^{2}$ \\ ${ }^{1}$ Yokohama Yamate Dog \& Cat Medical Center, 27-4 Kashiwaba Naka-ku Yokohama, Kanagawa, Japan 231-0866 \\ ${ }^{2}$ Tokyo Veterinary Cardiology Center, 8-9-12 Izumi Building 2F, Fukazawa, Setagaya, Tokyo 158-0081, Japan \\ ${ }^{3}$ Department of Small Animal Clinical Sciences, School of Veterinary Medicine, Rakuno Gakuen University, 582 Bunkyodai- \\ Midorimachi, Ebetsu, Hokkaido 069-8501, Japan
}

Correspondence should be addressed to Mitsuhiro Isaka; m-isaka@rakuno.ac.jp

Received 9 November 2020; Revised 23 December 2020; Accepted 19 January 2021; Published 27 January 2021

Academic Editor: Isabel Pires

Copyright (c) 2021 Ryuji Araki et al. This is an open access article distributed under the Creative Commons Attribution License, which permits unrestricted use, distribution, and reproduction in any medium, provided the original work is properly cited.

Isolated cleft of the anterior mitral leaflet (ICAML) in dogs without a septal defect is a rare pathological condition. Until now, only one paper has contributed to the detailed understanding of canine ICAML. Reports have confirmed that 3-dimensional echocardiography (3-DE) is a simple and fast imaging technique that is useful for the diagnosis of ICAML and morphological evaluation of the mitral valve in humans. However, to our knowledge, no studies have provided details about the effectiveness of 3-DE in ICAML diagnosis in dogs. Thus, we aimed to determine the usefulness of a diagnostic technique using 3-DE in a 2year-old Cavalier King Charles Spaniel with ICAML that exhibited mild mitral valve regurgitation. ICAML was initially assessed by transthoracic two-dimensional echocardiography. A diagnosis of congenital mitral regurgitation due to ICAML and understanding of the morphological structure of the valve was established based on the 3-DE findings.

\section{Introduction}

Isolated cleft of the anterior mitral leaflet (ICAML, not associated with atrioventricular septal defect) is a very rare cause of congenital mitral regurgitation (MR) in humans [1-3]. Only one case has been reported on the occurrence of ICAML in dogs [4]. Until now, a clear prognosis of the disease and the need for long-term comprehensive care have not been clarified. However, there are some studies in humans that have explored the application value of 3dimensional echocardiography (3-DE) in the diagnosis of ICAML and morphological evaluation of the mitral valve in humans $[5,6]$. Therefore, this case study is aimed at exploring the application value of 3-DE in the diagnosis of ICAML in dogs.

\section{Case Presentation}

The patient was a 2-year-old castrated male Cavalier King Charles Spaniel that weighed $8.2 \mathrm{~kg}$. The patient had a left ventricular systolic murmur confirmed at another hospital at 4 months of age. MR was confirmed using color-flow Doppler instead of two-dimensional echocardiography (2$\mathrm{DE})$. The patient was diagnosed with MR due to mitral valve dysplasia. The patient had been receiving temocapril hydrochloride $(0.12 \mathrm{mg} / \mathrm{kg} / \mathrm{SID})$ and pimobendan $(0.3 \mathrm{mg} / \mathrm{kg} / \mathrm{BID})$ from the age of 10 months. The patient came to our hospital for cardiac scrutiny.

On presentation to the hospital, the animal showed a heart rate of 147 beats per minute, a blood pressure of $117 / 80 \mathrm{mmHg}$, and oxygen saturation of $96 \%$. A grade IV/VI 


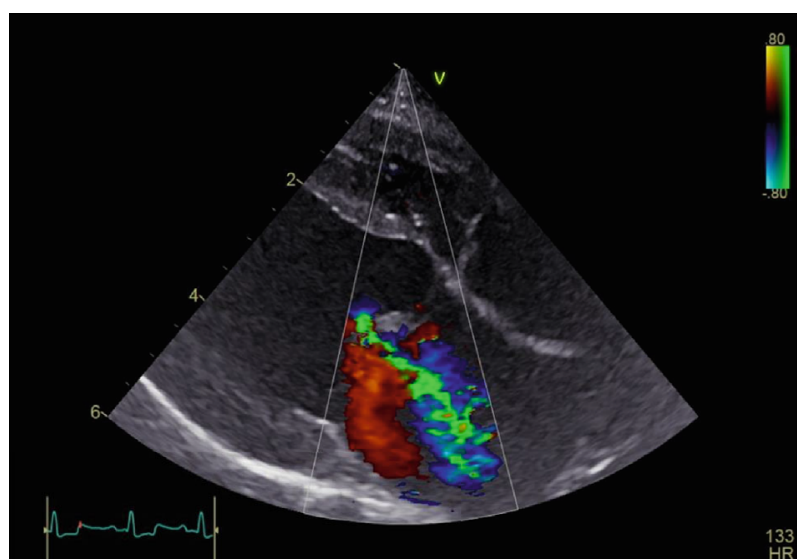

FIGURE 1: Color-flow Doppler echocardiogram obtained in the right parasternal long-axis view during systole. Mitral valve regurgitation is visible.

left apical systolic murmur was heard on auscultation. There were no symptoms such as coughing or exercise intolerance, and no abnormal findings were found in blood cell counts or biochemical tests. Normal levels of cardiac biomarkers, namely, $\mathrm{N}$-terminal probrain natriuretic peptide $(655 \mathrm{pmol} / \mathrm{dL}$; standard value: $<900 \mathrm{pmol} / \mathrm{dL})$, atrial natriuretic peptide $(14.5 \mathrm{pg} / \mathrm{mL}$; standard value: $8.6-105.8 \mathrm{pg} / \mathrm{mL}$ ), and cardiac troponin I $(0.012 \mathrm{ng} / \mathrm{mL}$; standard value: $<0.129 \mathrm{ng} / \mathrm{mL})$, were observed.

Thoracic radiography revealed a vertebral heart scale of $10.1 \mathrm{v}$ (standard value: $10.6 \pm 0.5 \mathrm{v}$ ) with no cardiac enlargement. No abnormal findings were found in the lung parenchyma or chest cavity, and there was no pulmonary vascular shadow. Electrocardiography revealed a sinus rhythm, and the electrical axis was $58^{\circ}$. Real-time 2-DE was performed using the ultrasound diagnostic equipment of Vivid E 95 (GE Healthcare, Japan). MR was observed on a left parasternal long-axis view using color-flow Doppler echocardiography (Figure 1). A cleft of the anterior leaflet of the mitral valve was observed from the left ventricular shortaxis view at the level of the mitral valve (Figure 2). There were no defects in the atrium or ventricular septum. To obtain a detailed view of the mitral morphology, real-time threedimensional (3-D) echocardiography (3-DE) was performed using a $4 \mathrm{v}$ probe. It directly showed the 3-D structure of the mitral valve cleft (MVC) in multiple views, revealing the position, shape, longitudinal diameter, and width, as well as the spatial position between the chordae tendineae surrounding the MVC and the aortic valve. The examination also revealed a maximum diameter of $2.1 \mathrm{~cm}$, a maximum of crack by cleft width of $0.8 \mathrm{~cm}$, and a V shape (Figure 3 ).

The blood flow velocity wave was measured by the continuous-wave Doppler method, which showed a maximum speed of $5.01 \mathrm{~m} / \mathrm{s}$ and a maximum pressure difference of $100 \mathrm{mmHg}$ in the mitral valve regurgitant flow velocity waveform. In the left ventricular inflow velocity waveform, the $\mathrm{E}$ wave and $A$ wave were 0.63 and $0.37 \mathrm{~m} / \mathrm{s}$, respectively. Morphological evaluation was performed using the left parasternal short-axis view. This revealed a left atrium-to-aorta ratio of 1.56 at the aortic valve level (standard value: $<1.6$ ), a left ventricular end-diastolic diameter of $33.0 \mathrm{~mm}$ at the papillary

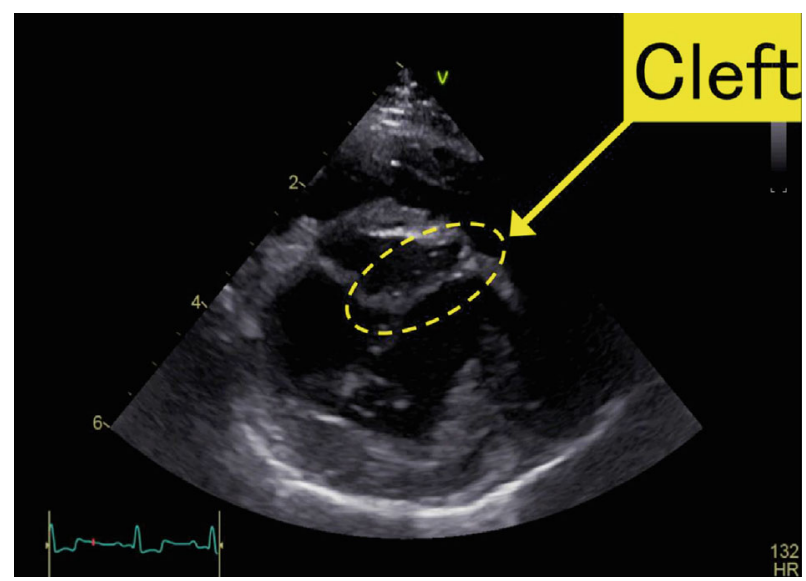

Figure 2: Two-dimensional echocardiography image. In the right parasternal short-axis view at the level of the mitral valve, a cleft is observed in the A2 segment of the anterior leaflet of the mitral valve.

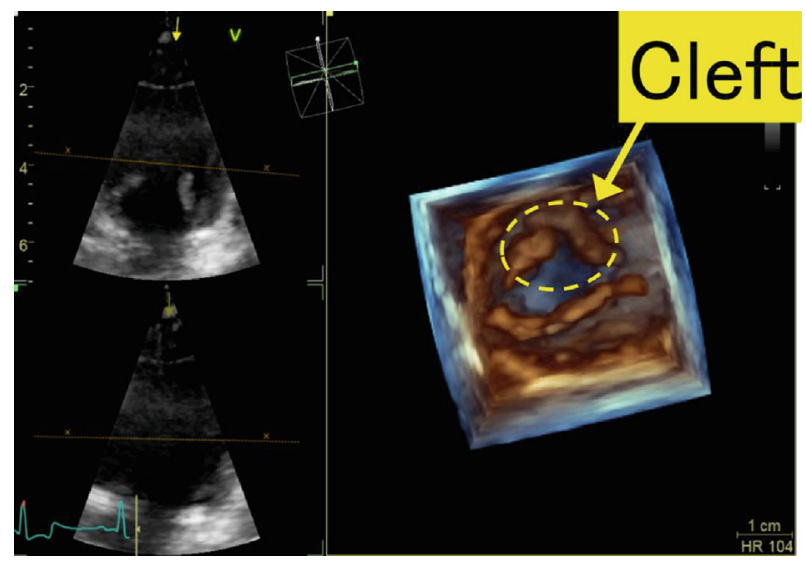

Figure 3: Three-dimensional echocardiographic image of the right parasternal short-axis view at the level of the mitral valve. A cleft of the anterior leaflet of the mitral valve can be clearly confirmed. The cleft is a $\mathrm{V}$ shape, with a maximum diameter of $2.1 \mathrm{~cm}$ and a maximum of crack by cleft width of $0.8 \mathrm{~cm}$.

muscle level, and the normalized left ventricular internal dimension in diastole $(1.67$; standard value: $<1.7)$. On the basis of these findings, we judged that the severity of the MR was mild.

The examinations led to a diagnosis of congenital MR due to ICAML. Although organic cardiac abnormality was observed, there was no heart enlargement (mitral valve disease was classified as Stage B1 per the American College of Veterinary Internal Medicine classification). Following discussion with the owner, treatment with oral temocapril hydrochloride and pimobendan, as prescribed from other hospitals, was continued. The symptoms related to the cardiac condition remained unchanged and did not worsen without treatment until the dog died of lymphocytic leukemia.

\section{Discussion}

Although anterior MVC can be encountered within the spectrum of atrioventricular septal defects (AVSD), it is rare 
for them to be diagnosed as an isolated finding in dogs. ICAML is assumed to be a partial defect of the endocardial bed [1-3]. In human medicine, the median duration from birth to diagnosis is reported to be 2 or 5 years $[1,3]$. In addition, among 2,177 patients with congenital heart disease, 22 (1\%) were reported to have isolated cleft mitral valve, and 5 cases were not related to diseases such as AVSD [3]. Depending on the severity of MR, surgical treatment such as direct suturing of the valve is indicated, and its prognosis has been proven to be good [6]. Complete diagnosis and structural evaluation of congenital mitral valve malformations using real-time 2-DE may be difficult because of the complicated 3 -D structure. Better morphometric and functional evaluations can be obtained with real-time 3-DE [7, 8]. It is important to measure the position and size of MVC in preparation for surgery, and real-time 3-DE is reportedly an important tool for cleft diagnosis and morphological mitral valve evaluation in humans $[5,6]$. In our case, although ICAML was observed on real-time 2-DE images, evaluation of the morphological structure, such as the exact position of the cleft and the size and thickness of the valve, was difficult. In terms of spatial visualization of MVC, real-time 3-DE enabled us to obtain information that could not be elucidated by real-time 2-DE, as observed in human medicine. Although MVC associated with AVSD in dogs has been reported [9], preoperative evaluation using real-time 3-DE has not been performed. Detailed mitral valve assessments using real-time 3-DE will be very useful for mitral valve surgery in dogs with or without AVSD.

The occurrence of ICAML in canines is rare and has only been reported once [4]. Thus, the present case is a very rare clinical case. In a case report by Otoni and Abbott, MVCinduced $\mathrm{MR}$ and left ventricular dynamic outflow tract obstruction were seen, but the symptoms related to the cardiac condition in this animal did not worsen until it died of lymphocytic leukemia, so they remained untreated [4]. Our patient did not show dynamic left ventricular outflow stenosis due to the cleft; however, symptoms could be worsened in the presence of acquired factors such as myxoma-like degeneration. Further studies involving careful follow-up with surgical treatment are required. Overall, this case study suggests that real-time 3-DE may be useful for the detailed and accurate diagnosis of ICAML in dogs.

\section{Conflicts of Interest}

The authors declare no conflict of interest in this study.

\section{References}

[1] D. Zhu, R. Bryant, J. Heinle, and M. R. Nihill, "Isolated cleft of the mitral valve clinical spectrum and course," Texas Heart Institute Journal, vol. 36, no. 6, pp. 553-556, 2009.

[2] A. Fraisse, T. A. Massih, B. Kreitmann et al., "Characteristics and management of cleft mitral valve," Journal of the American College of Cardiology, vol. 42, no. 11, pp. 1988-1993, 2003.

[3] A. El hammiri, A. Drighil, and S. Benhaourech, "Spectrum of cardiac lesions associated with isolated cleft mitral valve and their impact on therapeutic choices," Arquivos Brasileiros de Cardiologia, vol. 106, no. 5, pp. 367-372, 2016.

[4] C. Otoni and J. A. Abbott, "Mitral valve dysplasia characterized by isolated cleft of the anterior leaflet resulting in fixed left ventricular outflow tract obstruction," Journal of Veterinary Cardiology, vol. 14, no. 1, pp. 301-305, 2012.

[5] X. Yuan, A. Zhou, L. Chen, C. Zhang, Y. Zhang, and P. Xu, "Diagnosis of mitral valve cleft using real-time 3-dimensional echocardiography," Journal of Thoracic Disease, vol. 9, no. 1, pp. 159-165, 2017.

[6] M. H. Miglioranza, D. Muraru, S. Mihaila, J. C. Haertel, S. Iliceto, and L. P. Badano, "Isolated anterior mitral valve leaflet cleft: 3D Transthoracic echocardiography-guided surgical strategy," Arquivos Brasileiros de Cardiologia, vol. 104, no. 5, pp. e49-e52, 2015.

[7] B. Nicole and R. M. Lang, "Quantitative echocardiographic assessment of native mitral regurgitation: two- and threedimensional techniques," Journal of Heart Valve Disease, vol. 20, no. 5, pp. 483-492, 2011.

[8] X. Zeng, R. A. Levine, L. Hua et al., "Diagnostic value of vena contracta area in the quantification of mitral regurgitation severity by color Doppler 3D echocardiography," Circulation: Cardiovascular Imaging, vol. 4, no. 5, pp. 506-513, 2011.

[9] S. GOYA, N. KANNO, K. TESHIMA, T. ANNDO, and T. FUJIOKA, "Surgery for partial atrioventricular septal defect with pulmonary hypertension in an adult dog," The Journal of Veterinary Medical Science, vol. 80, no. 7, pp. 1183-1189, 2018. 